ANNALES

POLONICI MATHEMATICI

XLVII (1986)

\title{
Sur les oscillations amorties d'une solution de l'équation différentielle à retard
}

\author{
par Z. Mikolajska (Kraków)
}

Résumé. Dans la présente note nous démontrons une théorème sur l'existence d'une solution $x(t)$ de l'équation différentielle à retard, oscillante et telle que $x(t) \rightarrow 0$ pour $t \rightarrow \infty$.

Considérons l'équation

$$
x^{\prime}(t)=F\left(t, x(t), x_{t}\right)
$$

où $x_{t}(s)=x(t+s)$ pour $-h \leqslant s \leqslant 0$ et la fonction $F(t, x, \varphi)$ satisfait aux hypothèses suivantes:

HYPOTHESES $\mathrm{H}$.

$1^{\circ} F(t, x, \varphi)$ est continue dans l'ensemble $D=\boldsymbol{R} \times \boldsymbol{R} \times C([-1,0], \boldsymbol{R})$.

$2^{\circ} F(t, x, \varphi)$ est croissante par rapport à $\varphi$, c'est-à-dire que

$$
\text { pour } \varphi(s) \leqslant \psi(s) \text { on a } F(t, x, \varphi) \leqslant F\left(t, x_{1} \psi\right) \text {. }
$$

$3^{\circ} F(t, x, \varphi)$ est décroissante par rapport à $x$, c'est-à-dire que

$$
F(t, \bar{x}, \varphi) \geqslant F(t, \bar{x}, \varphi) \text { pour } \bar{x} \leqslant \bar{x} .
$$

$4^{0} x(t) \equiv 0$ est la solution unique de léquation (1) avec la condition initiale $x(t)=0$ pour $-h \leqslant t \leqslant 0$, c'est-à-dire

$$
F(t, 0,0) \equiv 0 \text {. }
$$
initiale

$5^{\circ}$ On admet l'unicité des solutions de l'équation (1) avec la condition

$$
x\left(t, t_{0}, \varphi\right)=\varphi\left(t-t_{0}\right) \quad \text { pour } t_{0}-h \leqslant t \leqslant t_{0} .
$$

$6^{\circ}$ Pour chaque $\varrho \neq 0$ on a

$$
\varrho \cdot F(t, \varrho, \varphi)<0 \text { pour } \varphi(s) \equiv \varrho \text { pour }-h \leqslant s \leqslant 0 .
$$

$7^{\circ}$ Pour chaque constante $\varrho, \mu$ on a

$$
\int_{0}^{\infty} F(s, \varrho, \mu) d s=K_{\varrho \mu}, \quad\left|K_{\varrho \mu}\right|<+\infty .
$$


THĖORĖME $T_{1}$. Sous les hypothèses $\mathrm{H}$ pour chaque solution $x(t)$ de l'équation (1), : :lle que $|x(t)| \leqslant \varrho<\infty$ pour $-h \leqslant t \leqslant 0, \lim _{t \rightarrow \infty} x(t)=\zeta$ existe, et $\xi$ est fini $(|\xi|<\infty)$.

Démonstration. I. Sous les hypothèses $1^{\circ}, 2^{\circ}$ et $5^{\circ}$ on a l'implication:

$$
\varphi \leqslant \psi \rightarrow x\left(t, t_{0}, \varphi\right) \leqslant x\left(t, t_{0}, \psi\right)
$$

(cf. Laksmikanthan, Leela [1]) et par suite, en vertu de $4^{\circ}$ pour $\varphi, \psi$ quelconques continues telles que $\varphi(s) \leqslant 0 \leqslant \psi(s)$ pour $-h \leqslant s \leqslant 0$ on a

$$
x(t, 0, \varphi) \leqslant 0 \leqslant x(t, 0, \psi) \text { pour } t \geqslant 0 .
$$

II. On vérifie facilement que

$$
|\varphi| \leqslant \varrho \rightarrow|x(t, 0, \varphi)| \leqslant \varrho \quad \text { pour } t \geqslant-h .
$$

Supposons que $x(t, 0, \varphi) \leqslant \varrho$ pour $t \leqslant t_{1}$

$$
x\left(t_{1}, 0, \varphi\right)=\varrho, \quad x(t, 0, \varphi)>\varrho \quad \text { pour } t_{1}<t \leqslant t_{1}+\varepsilon .
$$

On a donc, en vertu de (6) et (2)

$$
x^{\prime}\left(t_{1}, 0, \varphi\right) \leqslant F\left(t_{1}, \varrho, \varrho\right)<0
$$

et par suite, en vertu de (12)

$$
\varrho<x(t, 0, \varphi)<\varrho=x\left(t_{1}, 0, \varphi\right) \text { pour } t_{1}<t \leqslant t_{1}+\varepsilon
$$

d'où il vient que $x(t, 0, \varphi) \leqslant \varrho$ pour $-h \leqslant t<\infty$.

D'une une façon analogue on obtient l'inégalité

$$
x(t, 0, \varphi) \geqslant-\varrho \text { pour }-h \leqslant t<\infty
$$

et, par suite, pour chaque $\varphi$ continue telle que $0 \leqslant \varphi \leqslant Q$ on a

$$
0 \leqslant x(t, 0, \varphi)=x(t) \leqslant \varrho
$$

et pour $0 \geqslant \psi \geqslant-\varrho$

$$
0 \geqslant y(t)=x(t, 0, \psi) \geqslant-\underline{o} .
$$

La fonction $F(t, x, \varphi)$ étant croissante par rapport à $\varphi$ et décroissante par rapport à $x$, on a

d’où

$$
F(t, \varrho, 0) \leqslant x^{\prime}(t)=F\left(t, x(t), x_{t}\right) \leqslant F(t, 0, \varrho)
$$

$$
\int_{t_{1}}^{t} F(s, \varrho, 0) d s \leqslant x(t)-x\left(t_{1}\right) \leqslant \int_{i_{1}}^{1} F(s, 0, \varrho) d s
$$

Les intégrales $\int_{0}^{x} F(s, \varrho, 0) d s$ et $\int_{0}^{\infty} F(s, 0, \varrho) d s$ étant convergentes pour chaque 
$\varepsilon>0$ il existe $t_{\varepsilon}>0$ tel que

$$
-\varepsilon \leqslant \int_{t_{1}}^{t} F(s, \varrho, 0) d s \leqslant x(t)-x\left(t_{1}\right) \leqslant \int_{t_{1}}^{t} F(s, 0, \varrho) d s \leqslant \varepsilon \quad \text { pour } t \geqslant t_{1}
$$

$\geqslant t_{\varepsilon}$

donc $\lim _{t \rightarrow \infty} x(t)=\xi$ existe, $|\xi| \leqslant \varrho$.

D'une façon analogue on montre que $\eta$ existe, $|\eta| \leqslant \varrho$ tel que $y(t) \rightarrow \eta$ pour $1 \rightarrow x$.

Envisageons $\sigma(s)$ quelconque, continue pour $-h \leqslant s \leqslant 0$, il existe $\varrho>0$ et deux fonctions telles que

$$
\varrho \geqslant \varphi(s) \geqslant 0 \geqslant \psi(s) \geqslant-\varrho \text { pour }-h \leqslant s \leqslant 0
$$

et

$$
\varphi(s) \geqslant \sigma(s) \geqslant \psi(s) \quad \text { pour }-h \leqslant s \leqslant 0
$$

Posons

$$
x(t)=x(t, 0, \varphi), \quad y(t)=x(t, 0, \psi), \quad z(t)=x(t, 0, \sigma) .
$$

On a

$$
x(t) \geqslant z(t) \geqslant y(t) \text { pour }-h \leqslant t
$$

et

$$
\varrho \geqslant x(t) \geqslant 0 \geqslant y(t) \geqslant-\varrho \quad \text { pour }-h \leqslant t<\infty .
$$

Nous avons démontré qu'il existe un couple $\xi, \eta$ tel que

$$
\lim _{t \rightarrow \infty} x(t)=\xi, \quad \lim _{t \rightarrow \infty} y(t)=\eta
$$

donc pour chaque $\varepsilon>0$ il existe un $t_{\varepsilon}>0$ tel que

$$
\begin{array}{ll}
x(t) \leqslant \xi+\varepsilon & \text { pour } t \geqslant t_{\varepsilon}>0, \\
y(t) \geqslant \eta-\varepsilon & \text { pour } t \geqslant t_{\varepsilon}>0 .
\end{array}
$$

En vertu de (20) et (21)

$$
F\left(t, x(t), y_{t}\right) \leqslant z^{\prime}(t)=F\left(t, z(t), z_{t}\right) \leqslant F\left(t, y(t), x_{t}\right),
$$

et, en vertu de (20),

$$
F(t, \xi+\varepsilon, \eta-\varepsilon) \leqslant z^{\prime}(t) \leqslant F(t, \eta-\varepsilon, \xi+\varepsilon) \text { pour } t \geqslant t_{\varepsilon}>0
$$

d'où

$$
\int_{\mathrm{t}}^{1} F(s, \xi+\varepsilon, \eta-\varepsilon) d s \leqslant z(t)-z(\tau) \leqslant \int_{\tau}^{t} F(s, \eta-\varepsilon, \xi+\varepsilon) d s .
$$

L'intégrale $\int_{0}^{\infty} F(s, \varrho, \mu) d s$ étant convergent pour chaque $\varrho, \mu$ il existe 
pour chaque $\varepsilon>0, T_{\varepsilon} \geqslant t_{\varepsilon}$ tel que

$$
\begin{array}{r}
-\varepsilon \leqslant \int_{\mathfrak{i}}^{\mathrm{t}} F(s, \xi+\varepsilon, \eta-\varepsilon) d s \leqslant z(t)-z(\tau) \leqslant \int_{\mathfrak{\tau}}^{t} F(s, \eta-\varepsilon, \xi+\varepsilon) d s \leqslant \varepsilon \\
\text { pour } t \geqslant \tau \geqslant T_{\boldsymbol{q}} .
\end{array}
$$

Ainsi nous avons établi l'existence d'un $\zeta,|\zeta| \leqslant \varrho$ tel que

$$
\lim _{t \rightarrow \infty} z(t)=\zeta \text {. }
$$

Comme $z(t)$ est quelconque, notre théorème $T_{1}$ est démontré.

ThÉORème $T_{2}$. Sous les hypothèses $H$ chaque solution $z(t)$ de léquation (1) oscillante autour de 0 tend vers zéro pour $t \rightarrow \infty$ (c'est-à-dire elle est amortie).

Dé monst r ation. Envisageons une solution $z(t)$ quelconque, oscillante autour de 0 . Il existe donc une suite $\left\{t_{n}\right\} \rightarrow \infty$, telle que $z\left(t_{n}\right)=0$ pour $n$ $=1,2, \ldots$ mais en vertu du théorème $T_{1}$

$$
0=\lim _{n \rightarrow \infty} z\left(t_{n}\right)=\lim _{t \rightarrow \infty} z(t)=\xi
$$

donc $\lim _{t \rightarrow \infty} z(t)=0$.

Remarque $R_{1}$. Les hypothèses $H$ sont satisfaites par exemple dans le cas où

$$
F(t, x, \varphi)=\alpha(t) \varphi(-1)-\beta(t) x
$$

et $\alpha(t), \beta(t)$ sont deux fonctions continues dans $-h \leqslant t<\infty$ telles que

$$
\beta(t)>\alpha(t)>0 \text { pour } t \geqslant 0
$$

et

$$
\int_{0}^{\infty} \beta(s) d s=k
$$

on a

$$
\int_{0}^{\infty} \alpha(s) d s=k<k
$$

Dans le cas envisagé l'équation (1) a la forme

$$
\begin{aligned}
& x^{\prime}(t)=\alpha(t) x(t-1)-\beta(t) x(t), \\
& \int_{0}^{\infty} F(s, \varrho, \mu) d s=\int_{0}^{\infty} \alpha(s) d s \varrho-\int_{0}^{\infty} \beta(s) d s \mu=k \varrho-k \mu=K_{Q \mu}, \\
& \left|K_{e \mu}\right| \leqslant k \varrho+k \mu<\infty, \quad \varrho F(t, \varrho, \varrho)=[\alpha(t)-\beta(t)] \varrho^{2}<0 .
\end{aligned}
$$

Du théorème $T_{2}$ il résulte que chaque solution oscillante de l'équation (1') converge vers zéro. 
Remarque $R_{2}$. Le théorème $T_{2}$ n'entraine pas l'existence d'une solution oscillante de l'équation (1). Par exemple dans le cas où

$$
F(t, x, \varphi)=-\beta(t) x
$$

chaque solution de (1) a la forme

$$
x(t, 0, \varphi)=\varphi(0) \exp \left[-\int_{0}^{t} \beta(s) d s\right.
$$

et par suite il n'existe pas de solution oscillantes autour de 0 (different de $x(t) \equiv 0)$ tendique dans le cas où $\int_{0}^{\infty} \beta(s) d s=k<\infty$

$$
\lim _{t \rightarrow \infty} x(t, 0, \varphi)=\varphi(0) e^{-k} \neq 0 \quad \text { est finie. }
$$

Remarque $R_{3}$. Dans le cas où

$$
F(t, x, \varphi)=\alpha(t-1) \varphi(-1)-\alpha(t) x
$$

où $0<\alpha(t-1)<\alpha(t)$ et $\int_{0}^{\infty} \alpha(t) d t=k<\infty$ chaque solution $x(t)$ de l'équation

$$
x^{\prime}(t)=\alpha(t-1) x(t-1)-\alpha(t) x(t)
$$

converge vers une limite finie pour $t \rightarrow \infty$ et chaque solution oscillante satisfait à la condition $\lim _{t \rightarrow \infty} x(t)=0$. D'après un théorème de $O$. Arino et $P$. Sérguier [2] (cf. le théorème $T_{34}$ ) chaque solution $x(t)$ telle que

$$
x(0)+\int_{-1}^{0} \alpha(s) d s=0
$$

est oscillante autour de 0 . De notre théorème $T_{2}$ il résulte donc que chaque solution de $\left(\mathrm{P}_{1}\right)$ satisfaisante à $(\mathrm{Q})$ tend vers zéro pour $t \rightarrow \infty$.

Remarque $R_{4}$. Il est évidente que l'hypothèse $6^{\circ}$ peut être remplacée par l'hypothèse $6^{\circ}$ : chaque solution $x\left(t, t_{0}, \varphi\right)$ de l'équation (1) est bornée (pour $t \geqslant t_{0}-h$ ).

Par exemple (cf. [3]) dans le cas où l'équation (1) a la forme

$$
x^{\prime}(t)=f(t-1, x(t-1))+g(t, x(t))
$$

où les hypothèses suivantes sont satisfaites.

HyPothéses A. $f(t, x)$ et $g(t, x)$ sont continues par rapport à $t, x$, $f(t, x)$ est croissante par rapport à $x$,

$$
x \cdot[f(t, x)+g(t, x)]<0 \quad \text { pour } x \neq 0, f(t, 0) \equiv 0
$$

et l'éqution (1) satisfait à l'unicité des solutions satisfaisant la condition initiale (5). On obtient donc dans le cas envisagé le Théorème $T_{2}$, 
admettons les Hypothèses B:

$1^{\circ} g(t, x)$ est décroissante par rapport à $x$,

$2^{\circ}$ pour chaque $\varrho, \mu$ on a

$$
\int_{0}^{\infty}[f(s-1, \varrho)+g(s, \mu)] d s=K_{e \mu}, \quad\left|K_{e \mu}\right|<\infty .
$$

Sous les hypothèses $\mathrm{A}$ et $\mathrm{B}$ chaque solution oscillante de l'équation (1) tend vers zéro.

De la remarque $R_{4}$ il s'ensuit que dans le cas de l'équation $\left(P_{1}\right)$ envisagé dans la remarque $R_{3}$ on peut remplacer l'inégalité (6) par

$$
0<\alpha(t), \quad \int_{0}^{\infty} \alpha(t) d t=k<\infty .
$$

\section{Rëférences}

[1] V. Laksmikanthan, S. Leela, Differential and integral inequalities, Vol. II. Academic Press. New York and London 1969.

[2] O. Arino et P. Sérguier, Thèse de doctorat d'état. Publications Mathématiques, Université de Pau et du Pays l'Adour (1980).

[3] Z. Mikołajska, Une remarque sur les solutions bornées d'une équation différentielle à retard, Ann. Polon. Math. 47 (1986), 105-114.

Reçu par la Rédaction le 1981.06.18 